\title{
Reconstructive processes in the free recall of categories
}

\author{
HOWARD R. POLLIO*, PATRICK MAHONEY, and MICHAEL J. GREEN \\ University of Tennessee, Knoxville, Tennessee 37916
}

\begin{abstract}
Three experiments, involving three different groups of Ss, were conducted. For each experiment, one group of Ss never saw the stimulus materials but produced "recall outputs" on the basis of someone else's description of the input list. A second group produced a description of, and then recalled, the list, while a third group recalled the list under standard conditions. Each experiment involved different materials: for Experiment I, exhaustive categories were used; for Experiment II, high-frequency category items were used; and for Experiment III, low-frequency items were used. Results showed all lists were reconstructible with the degree of reconstructibility following the order of experiments. An examination of descriptions produced indicated significant differences between those leading to lengthy outputs and those leading to less extensive outputs; basically, these differed in terms of the degree to which specific information was or was not present in the description. Results were discussed in terms of Bartlett's theory of remembering.
\end{abstract}

How much of a S's recall output is reconstructive? It seems strange that this question still has to be asked roughly 40 years after Bartlett's classic treatment of the subject in 1932. The fact remains, however, that despite tremendous recent emphasis on retrieval processes in recall, only a small number of investigators have considered the possibility that, in many cases, reconstructive rather than more strictly retrieval processes may account for what, and how much, is recalled. The reason such processes have not been stressed probably has to do with difficulties involved in measuring schemata, or those mental structures described by Bartlett as the basis on which reconstructive recall is accomplished.

In Bartlett's original description (1932), a schema was characterized as an "active mass of organized past reactions in experience ... [where] each new group of experiences [does not persist] as an isolated member of some passive patchwork [but rather must] be regarded as constituents of living, momentary settings belonging to the organism, or to whatever parts of the organism are concerned in making a response of a given kind [p. 201]." For this approach, the representation of experience is seen as a dynamic event and, further, as dynamically interacting with both $\mathrm{S}$ and task demands at a given moment in time. Individuals remembered in accordance with their schemata, where such schemata took account of the specific requirements imposed by a recall task. Depending on the task, an individual's recall was more or less improvised-reconstructed-in accordance with his understanding of what was to be done and how he could do it given the constraints imposed by task and situation.

The purpose of the present experiment is to provide some measure of mnemonic schemata and, further, to describe how they function in bringing about the performance we call recall. In this experiment, one group of Ss never saw a list of to-be-recalled items; they

*This paper was written while the senior author was in residence at the University of Guelph, Guelph, Ontario, Canada. simply produced "recall records" on the basis of someone else's description of stimulus materials. A second group of Ss produced both an initial description of their experiences and then recalled the list, while a third group simply recalled the list. Since we were also interested in assessing these procedures over different types of materials, three different experiments, each involving all three groups, were conducted. The only difference in each experiment was the type of material used: for Experiment I, exhaustive category items were presented; for Experiment II, high-frequency category exemplars were presented; and for Experiment III, low-frequency exemplars were presented. In all cases, our primary question was: how much reconstruction is there in category recall?

\section{METHOD}

Since all three experiments differed only in the materials used, the experimental procedure will be described in detail for the first experiment only. The results for all three experiments will also be presented at the same time.

\section{Procedures}

Three groups of Ss were used in each experiment. The initial instructions given to $S$ s were standard free recall instructions, i.e., Ss would see a list of words presented by means of a slide projector and then would be asked to write down their recall. One group of Ss-the recall-only group-was shown the list of words at a $2-\mathrm{sec} /$ word rate and then was asked to write down as many words as they could at the conclusion of three presentations of the entire list. A second group of Ss-the describe-recall group-was shown the list of words, again at a 2-sec rate, and then asked to describe the list in writing so that another person would "know what words were contained in the list without listing the specific words just seen." No further instructions were given to $S s$ in this group. Although $S s$ were allowed as much time as they needed, no $S$ actually took longer than about $5 \mathrm{~min}$. The $\mathrm{Ss}$ in this group wrote out their descriptions and, following this, wrote down all the words they could remember from the previously presented list. A third group-the reconstructive group--never saw any list of stimulus words. Each $S$ in this group was simply presented with the description written by one $S$ in the describe-recall group and was then asked to reconstruct the list from the description alone. All 
Table 1

Mean Number of Words Recalled by All Groups for All Three Experiments

\begin{tabular}{|c|c|c|c|c|c|c|c|}
\hline \multirow{3}{*}{$\begin{array}{c}\text { Experi- } \\
\text { ment }\end{array}$} & \multicolumn{6}{|c|}{ Group } & \multirow{3}{*}{$\begin{array}{c}\text { Maximum } \\
\text { Possible } \\
\text { Recall }\end{array}$} \\
\hline & \multicolumn{2}{|c|}{ Recall Only } & \multicolumn{2}{|c|}{ Describe-Recall } & \multicolumn{2}{|c|}{ Reconstructive } & \\
\hline & Mean & SD & Mean & $\mathrm{SD}$ & Mean & $\mathrm{SD}$ & \\
\hline I & 17.76 & 3.28 & 17.64 & 2.36 & 14.88 & 3.55 & 20 \\
\hline II & 20.95 & 3.11 & 21.25 & 2.51 & 13.50 & 1.83 & 25 \\
\hline III & 17.65 & 3.25 & 15.60 & 3.56 & 4.45 & 4.15 & 25 \\
\hline
\end{tabular}

Ss were tested individually, with each $S$ in the reconstructive group being paired with a given $S$ in the describe-recall group. All pairings between $S s$ in these two groups were randomly done; no attempt was made to match Ss on any specific basis.

The Ss in all groups were students at the University of Tennessee. The total number used in the first experiment was 75 , with these Ss being randomly distributed into groups of 25 each. In Experiment II, 60 Ss were used, 20 per group, and in Experiment III, $60 \mathrm{Ss}$ were used, again, 20 per group. In these latter two experiments, all Ss were assigned on the basis of a random procedure.

\section{Stimulus Materials}

The 20 words used in Experiment I involved 4 words from each of five different exhaustive categories. Items were taken from the Cohen, Bousfield, and Whitmarsh norms (1957). The exhaustive categories used in Experiment I were: (1) grade levels in college (freshman, sophomore, junior, senior); (2) immediate family members (father, mother, sister, brother); (3) face cards in a deck of playing cards (jack, queen, king, ace); (4) seasons of the year (spring, summer, autumn, winter); and (5) basic arithmetic operations (addition, subtraction, multiplication, division). All items were presented in random order for three presentations of the entire list.

The stimulus materials used in Experiment II involved 25 words. These 25 words were chosen as stimuli from five different categories, again taken from the Cohen, Bousfield, and Whitmarsh norms. These were selected to be of high frequency as responses to category names, and because of the positive correlation between frequency of a word as a category exemplar and general frequency of occurrence in the language, stimulus words were of high occurrence frequency as well. The categories and stimulus words used were (1) sports (baseball, football, hockey, tennis, golf); (2) men's names (Joe, John, Bill, Tom, Bob); (3) fruits (apple, peach, pear, grape, banana); (4) countries (England, France, Germany, Russia, Spain); and (5) colors (red, brown, green, yellow, blue). Of the 25 words, 15 were of $A$ frequency or better according to the Thorndike-Lorge count, while 24 of 25 were of greater frequency than 15 times per million. As before, the list had three randomized repetitions of all 25 words, making a total of 75 items in all.

The stimulus materials used in Experiment III again involved 25 words, including 5 words from each of five categories, and again these items were taken-both categories and exemplars-from the Cohen, Bousfield, and Whitmarsh norms. These items were selected to be of low frequency of occurrence as responses to category names, and again, as a result, of low frequency of occurrence in the language. The categories and stimulus words used were: (1) animals (weasel, leopard, camel, donkey, woodchuck); (2) vegetables (cabbage, spinach, turnip, pumpkin, carrot); (3) jobs or professions (dentist, printer, chemist, blacksmith, grocer); (4) musical instruments (clarinet, cello, piccolo, trumpet, fiddle); and (5) men's names (Adam, Simon; Wallace, Howard, Bernard). Of these 25 words, none were of A frequency or better and only 6 were of greater than 15 per million frequency. As in Experiment II, the list had three randomized repetitions of the 25 words, making a total of 75 in all.

\section{General Summary of Procedure}

All three experiments involved three groups of $S_{s}$ : a recall-only group, a describe-recall group, and a reconstructive group. In Experiment I, there were four items from each of five exhaustive categories; in Experiment II, there were five high-frequency exemplars from each of five nonexhaustive categories; and in Experiment III, there were five low-frequency exemplars from each of five nonexhaustive categories.

\section{RESULTS}

\section{Number of Items in Recall}

Table 1 presents the number of items recalled for each of the three groups for each of the three experiments. It should be kept in mind that the maximum possible recall in Experiment I was 20 items, while in Experiments II and III, it was 25 for each of the three groups.

Looking first at Experiment I, we can see that of the 20 possible items, the recall-only and describe-recall groups recalled almost 18 of these items. The major exception in both cases was that Ss did not produce the word autumn but, rather, often incorrectly responded with the word fall. The data also show that the reconstructive group recalled about 15 items. Taking the reconstructive group as a proportion of the recall-only group, we.can see that Ss in the reconstructive group produced recalls $84 \%$ as extensive as those produced by groups experiencing more standard recall situations.

An analysis of variance performed over the data contained in the first row of Table 1 showed significant differences among the three groups $[\mathrm{F}(2,72)=11.68$, $p<.01]$. The results for the high-frequency items are presented in the second row of the table, where it is important to keep in mind that the maximum possible recall is 25 items. An examination of the results produced by the three groups again showed little or no difference between the recall-only and describe-recall groups, whereas both were significantly better than the reconstructive group $[\mathrm{F}(2,57)=31.71, \mathrm{p}<.01]$. The important thing to note, however, is that under these conditions, the reconstructive group still produced an output containing 13.5 words, or roughly $64 \%$ of the number of items recalled by the group given standard recall instructions. If we look now at the third row of the table, we can see that results are somewhat different for the low-frequency recall items. Here $S s$ in the recall-only group and the describe-recall group produced different average recalls. In addition, the reconstructive 
group produced an output of only 4.5 items, or only $24 \%$ of the recall-only output. An analysis of variance done over these data produced an $F$ value $(\mathrm{df}=2,57)$ of $106.07(\mathrm{p}<.01)$, and on the basis of a $t$ test $(t=2.10$, $\mathrm{df}=59, \mathrm{p}<.05$ ), results showed a significant difference between the recall-only group and the describe-recall group.

\section{Nature of Descriptions Produced by Describe-Recall Group}

Since one purpose of the present experiment was to provide some assessment of mnemonic schemata, it is of interest to examine the descriptions produced by $\mathrm{Ss}$ in the describe-recall group. A preliminary examination of these data showed that the mean number of words in the exhaustive category case was 70.0 (median $=40.0$ ), in the high-frequency case was 56.4 (median $=36.5$ ), and in the low-frequency case was 69.2 (median $=43.5$ ). Due to unequal variances, however, an analysis of variance computed over these data showed no significant difference $(F<1.00)$ among mean values, even when values were normalized. If, however, we look at the ratio of words recalled to number of words in the description, it is quite clear that each word in the description was more "efficient" in Experiment I than in either Experiment II or Experiment III. That is, it took a median value of about 40 words to specify 15 words in Experiment I, 13.5 in Experiment II, and 4.5 in Experiment III.

Given these data, it is of interest to correlate the number of words in the description with the number of words recalled by a given $\mathrm{S}$ in the describe-recall group. Here correlations are suggestive but not unequivocal; the product-moment correlations between the number of words in the descriptions produced by the describe-recall Ss and the number of words recalled by these Ss were $-.92(\mathrm{df}=24, \mathrm{p}<.01)$ for exhaustive categories and $-.04(\mathrm{df}=19, \mathrm{p}>.10)$ for both other lists. The correlations between description length and the number of words recalled by paired $S s$ in the reconstructive group produced values of $.30(\mathrm{df}=24, \mathrm{p}>.05)$ for exhaustive categories, $.06(\mathrm{df}=19, \mathrm{p}>.10)$ for high-frequency items, and $.77(\mathrm{df}=19, \mathrm{p}<.01)$ for low-frequency items. What this means is that, although there were no differences in description length among the three groups, the pattern of correlations was different for the describe-recall and reconstructive groups. Basically, these results show that the longer the description, the less the recall of exhaustive category items for Ss in the describe-recall group, and the longer the description, the greater the recall for paired $\mathrm{Ss}$ in the reconstructive group in Experiment III.

From a theoretical point of view, probably the most important correlations are those between the number of items appearing in recall for the describe-recall and reconstructive groups. Results here reveal a consistent pattern, namely, that correlations between the number of items recalled by paired Ss in the describe-recall and reconstructive groups decreased over each of the three lists. Thus, for Ss exposed to exhaustive categories, the correlation was $.59(\mathrm{df}=24, \mathrm{p}<.01)$; for high-frequency items (Experiment II), the correlation was $.40(\mathrm{df}=19, \mathrm{p}<.05)$; and for low-frequency items (Experiment III), the correlation was $.12(\mathrm{df}=19$, p>.10).

Since one of our major concerns was in describing the characteristics of schemata used by Ss in effecting recall, a content analysis of all description protocols was undertaken for each of the three experiments. In addition, recall outputs for reconstructive Ss for all three lists were broken at the median such that effective descriptions-those leading to lengthy recalls-could be separated from those that led to relatively short recalls. An examination of protocols for Ss in all three groups suggested that five properties would describe these adequately, and once these were identified, the frequency of occurrence for each was determined for both short and long recall outputs.

The first feature recovered from the data concerns category structure either specifically by name, such as by using the word we had used in getting items from the Cohen, Bousfield, and Whitmarsh norms, or implicitly, by listing all items of a given category together and only at the end of this listing saying, "These are all fruits," "These are all sports," etc. A second feature recovered from the data concerned a listing of all five categories. In all cases, this meant that $S$ either listed all five categories explicitly or said somewhere in the description that there were five categories in toto. A third feature that was present to a greater or lesser degree was an explicit or implicit statement as to the number of items in a category. So, for example, in Experiment I, this feature would occur in the description as, "There are four items in each category." In Experiments II and III, Ss often gave a statement of the form: "There are five items per category." A fourth feature is what we will call within-category distinguishers. This was an attempt by Ss in the describe-recall group to provide a very specific definition for one or more items. In the case of sports, for example, some Ss wrote: "We are talking about the category of sports and there are five sports. One of these sports is played on ice and involves using a puck Another one involves using an oval-shaped ball and where the players use shoulder pads. .." Ss made similar descriptions for all categories. Finally, some small number of Ss simply provided a list of distinguishers without making use of any category structure at all, i.e., where the description essentially provided a noncategorized list of definitions. In general, this was a very infrequently used approach.

Table 2 presents the relative frequency of occurrence of each of these five features for each list separately. In addition, these data are divided into two sets-high and low-where high sets involve records for $S$ in the reconstructive group who produced recalls above the 
Table 2

Properties of Descriptions Written by Describe-Recall Subjects and Their Use by High and Low-Recall Subjects in Reconstructive Groups

\begin{tabular}{|c|c|c|c|c|c|c|}
\hline \multirow[b]{2}{*}{ Descriptive Feature } & \multicolumn{2}{|c|}{ Experiment I } & \multicolumn{2}{|c|}{ Experiment II* } & \multicolumn{2}{|c|}{ Experiment III* } \\
\hline & $\begin{array}{c}\text { High } \\
(\mathrm{N}=10)\end{array}$ & $\begin{array}{c}\text { Low } \\
(\mathrm{N}=10)\end{array}$ & $\begin{array}{c}\text { High } \\
(\mathrm{N}=8)\end{array}$ & $\begin{array}{c}\text { Low } \\
(\mathrm{N}=7)\end{array}$ & $\begin{array}{c}\text { High } \\
(N=6)\end{array}$ & $\begin{array}{c}\text { Low } \\
(\mathrm{N}=10)\end{array}$ \\
\hline 1. Category Structure & 10 & 7 & 8 & 6 & 5 & 10 \\
\hline 2. Listing of All Five Categories & 9 & 2 & 8 & 5 & 5 & 9 \\
\hline 3. Number of Items in Category & 10 & 3 & 2 & 1 & 3 & 1 \\
\hline 4. Distinguishers Within Categories & 2 & 0 & 6 & 1 & 5 & 0 \\
\hline 5. List of Distinguishers & 0 & 3 & 0 & 1 & 1 & 0 \\
\hline
\end{tabular}

*There are an unequal number of $S$ s in high and low groups primarily because scores tied at the median were not considered in the present analysis.

median and where low sets involved Ss who produced recalls below the median. In the case of Experiment I, there were $10 \mathrm{Ss}$ above and 10 below the median, with 5 falling exactly at the median. In the case of Experiment II, there were 8 Ss above the median and 7 below, while in Experiment III, there were 6 above and 10 below.

The most important aspects of Table 2 concern differences between lists and between high and low outputs. For example, in the case of exhaustive categories, the major difference between high and low output protocols concerns the presence or absence of a specific entry for the number of items in the category (Feature 3) and a specific listing of all five categories (Feature 2). An examination of the data for Experiment II indicates that the major difference between high and low recall ouputs was in terms of the presence or absence of distinguishers (Feature 4). This same pattern, emphasized perhaps even more strongly, also prevails in describing differences between high and low protocols in Experiment III.

On the basis of these results, we can say that the most common elements in descriptions producing "good" recalls for exhaustive categories involved category structure, number of words in a category, and list of all five categories. For $S s$ in the reconstructive group producing short recalls, such descriptions included some statement as to category structure and very little else. For high-frequency category items, the most common descriptive features producing "good" recalls involved category structure, a listing of all five categories, and a specific description of the distinguishing features for each element in the categories. For "poor" recalls, only two properties were used: category structure and a listing of all five categories. Good recall for low-frequency category items was accomplished by presenting not only category structure, but also distinguishers for all items. In contrast, poor recall Ss were presented with category structure and told that there were five categories but were never presented with specific item distinguishers.

\section{DISCUSSION}

The results of the present study suggest that how much of a S's recall output is reconstructive depends on a number of factors, the most important of which in the present context have to do with the type of materials presented. More specifically, these results indicate that almost all (84\%) of S's output may be reconstructive for exhaustive categories and that only some of his output (24\%) may be reconstructive for low-frequency category items. These conclusions are based on the relative degree of "recall" exhibited by Ss in the reconstructive group-Ss who were never exposed to stimulus items but simply produced "recall" in response to a description of the stimulus list.

On this basis, it seems reasonable to propose that for certain types of highly regular materials such as exhaustive categories, remembering is probably best characterized as a constructive act within which individual items are probably never stored in the first place. With less reconstructible materials, such as the materials used in List III, Ss seem to depend more and more on specific cues to retrieve, rather than reconstruct, uniquely correct items. This is particularly true in the context of an artificial situation such as is provided by a laboratory experiment where an item is counted correct if and only if the exact word is recalled. Within such task constraints, it is obvious that the successful $\mathrm{S}$ must change from a reconstructive strategy such as might have been used in Experiment I to a more detailed and retrieval-oriented approach such as seems to have been used in Experiment III.

The overall conclusion, then, is that recall involves more than simply retrieval or reconstructive processes; rather, it must involve a whole series of decisions, with the most important of these having to do with how recall is to be accomplished in a particular setting. In the case of category materials, the viable options seem to boil down to whether or not the $S$ will remember (1) simply the category name, (2) the category name plus some small number of properties about the category, or (3) the category name and a great many specific details about individual category items. The more general point to be kept in mind, however, is not the specific structure of the strategy used, but rather that the process of recall is always embedded within a total matrix of behavior and that the human $S$ is continuously making decisions as to how to go about 
recall, given the constraints of a particular situation and S's understanding of his task in that situation.

This suggests that neither process-reconstruction or retrieval-by itself can account for how Ss recall different types of materials. If we return to Bartlett's (1932) analysis of remembering, however, it is clear that retrieval processes represent a more restricted class of mnemonic operations than do reconstructive ones. By this, we simply mean that during the course of hearing or seeing a list, $\mathrm{S}$ first makes a decision as to whether or not to store a vague and highly abstracted description of the input or to attempt a highly specific description of some or all items. The former case would seem to apply to highly structured materials such as exhaustive categories and, to a lesser degree, to high-frequency category exemplars; the latter would seem to apply to the case of low-frequency items where more details are needed to cue the retrieval of an exact recall output.

The present analysis also has some implications for the continuing debate (see earlier discussions by Pollio \& Gerow, 1968; Slamecka, 1972) between associative and cognitive interpretations of category clustering. Probably the most important of these is that two experimental and theoretical traditions are at issue here and that much, if not all, of the data gathered in support of one or the other position is open to reinterpretation by the opposite point of view.

What present results do suggest in regard to this controversy is that what you ask a $\mathrm{S}$ to do strongly affects how he does it and that different materials and/or tasks seem to evoke different approaches, and thereby to suggest different theoretical mechanisms. If this is true, then the use of a list of associated items (see Jenkins, Mink, \& Russell, 1958; Pollio, Kasschau, \& DeNise, 1968) will produce results commensurate with the view that Ss cluster words in recall to the degree that they share associative relations, while the use of exhaustive categories (Cohen, 1963a, b), rule-governed materials (Pollio, Linder, \& Foote, 1973), or blocked presentation of lower-frequency category items (Cofer, Bruce, \& Reicher, 1966; Slamecka, 1972) will produce results commensurate with a constructive description such as implied by Bartlett. Perhaps this means that, as Marton (1970) put it: "One of the most general findings of psychology is that human beings when acting as subjects ... kindly adjust themselves to experimental demands .... and very efficiently prevent the persistence of any general theory of mind [p. 87]."

Alternately, these data might mean that a unified theory of memory--like a general theory of mind-is not yet possible and that all theoretical interpretations must, of necessity, remain specific to the specific combinations of materials, conditions, and Ss used in any given study. Such an approach, of course, is quite congenial to Bartlett's position on remembering, a position which stresses process rather than preexisting or newly established structural connections between items as crucial to the process of recall.

\section{REFERENCES}

Bartlett, F. C. Remembering. Cambridge, England: Cambridge University Press, 1932.

Cofer, C. N., Bruce, D. R., \& Reicher, G. M. Clustering in free recall as a function of certain methodological variations. Journal of Experimental Psychology, 1966, 71, 858-866.

Cohen, B. H. An investigation of recoding in free recall. Journal of Experimental Psychology, 1963a, 65, 368-376.

Cohen, B. H. Recall of categorized word lists. Journal of Experimental Psychology, 1963b, 66, 227-234.

Cohen, B. H., Bousfield, W. A., \& Whitmarsh, G. A. Cultural norms for verbal items in 43 categories. Technical Report No. 22, Contract No. 631(000), Office of Naval Research and University of Connecticut, 1957.

Jenkins, J. J., Mink, W. D., \& Russell, W. A. A ssociative clustering as a function of verbal association strength. Psychological Reports, 1958, 4, 127-136.

Marton, F. Structural dynamics of learning. Stockholm: Almqvist \& Wiksell, 1970.

Pollio, H. R., \& Gerow, J. R. The role of rules in recall. American Journal of Psychology, 1968, 81, 303-313.

Pollio, H. R., Kasschau, R. K., \& DeNise, H. Associative structures and the temporal characteristics of free recall. Journal of Experimental Psychology, 1968, 76, 190-197.

Pollio, H. R., Linder, W., \& Foote, R. A. Process-governed changes in the temporal properties of ordered recalls. Scandinavian Journal of Psychology, 1973, 14, 114-120.

Slamecka, N. J. The question of associative growth in the learning of categorized material. Journal of Verbal Learning \& Verbal Behavior, 1972, 11, 324-332.

(Received for publication December 5, 1972; revision received July $12,1973$. ) 